\title{
Stability of Designed Proteins against Mutations
}

\section{Citation}

Broglia, R. A., G. Tiana, H. E. Roman, E. Vigezzi, and E. Shakhnovich. 1999. “Stability of Designed Proteins against Mutations." Physical Review Letters 82 (23): 4727-30. https://doi.org/10.1103/ physrevlett.82.4727.

\section{Permanent link}

http://nrs.harvard.edu/urn-3:HUL.InstRepos:41417277

\section{Terms of Use}

This article was downloaded from Harvard University's DASH repository, and is made available under the terms and conditions applicable to Other Posted Material, as set forth at http:// nrs.harvard.edu/urn-3:HUL.InstRepos:dash.current.terms-of-use\#LAA

\section{Share Your Story}

The Harvard community has made this article openly available.

Please share how this access benefits you. Submit a story.

Accessibility 


\title{
Stability of Designed Proteins Against Mutations
}

\author{
R. A. Broglia ${ }^{1,2,3}$, G. Tiana ${ }^{3,1}$, H. E. Roman ${ }^{1,2}$, E. Vigezzi ${ }^{1,2}$ and E. Shakhnovich ${ }^{4}$ \\ ${ }^{1}$ Dipartimento di Fisica, Università di Milano, Via Celoria 16, I-20133 Milano, Italy. \\ ${ }^{2}$ INFN, Sezione di Milano, Via Celoria 16, I-20133 Milano, Italy. \\ ${ }^{3}$ The Niels Bohr Institute, University of Copenhagen, 2100 Copenhagen, Denmark. \\ ${ }^{4}$ Department of Chemistry and Chemical Biology, Harvard University, 12 Oxford Street, \\ Cambridge, MA 02138
}

(April 19, 2018)

\begin{abstract}
The stability of model proteins with designed sequences is assessed in terms of the number of sequences (obtained from the designed sequence through mutations), which fold into the "native" conformation. By a complete enumeration of the total number of sequences obtained by introducing up to 4 point mutations and up to 7 composition-conserving mutations (swapping of amino acids) in a 36 mers chain, it is found that there are $10^{8}-10^{9}$ sequences which in the folding process target onto the "native" conformation. Consequently, proteins with designed sequences display a remarkable degree of stability and, to a large extent, of designability.
\end{abstract}


A number of previous analyses [1 [6] (reviewed in [0,8]) have provided arguments and supporting evidence for the deep connection existing between the energetic properties of protein sequences and their ability to fold fast into their native conformations. In particular it was found [2 [4] that the presence of a large (compared to the dispersion of interaction energies) energy gap between the native state and the bulk of misfolded conformations that are structurally dissimilar to the native state is an important factor that ensures fast folding into the native conformation (foldability requirement). A number of observations support the notion that sequences of natural proteins have been optimized to satisfy the foldability requirement:

1) Random sequences undergo non-cooperative folding transition [9, 10] while designed sequences and proteins fold cooperatively [1, [3, $4,11,12]$.

2) The native state of random sequences is very unstable even to small changes in potential function [13] while the ones that have larger gaps are much more robust with respect to changes in the energy function [14,15]. The latter behavior is characteristic of real proteins that exhibit the remarkable ability to maintain their native structure intact in a wide range of conditions including variation of temperature, $\mathrm{pH}$, solvent composition etc.

3) It was shown theoretically that ground (native) states of random sequences are very unstable with respect to point mutations: the probability that a mutated sequence has the same native state scales as $\gamma^{-8}$ where $\gamma$ is the number of conformations per one residue in the chain [16]. In contrast, real proteins are able to accomodate numerous mutations that are neutral with respect to structure changes [17]. This fact has obvious implication for the molecular evolution of proteins: it accounts for the existence of large families of proteins that may have diverged from a common root. Proteins belonging to a family have homologous sequences and their native states are structurally similar.

While the stability of designed sequences with respect to point mutations has been demonstrated [18] in simulations, and the fact that larger energy gaps imply greater ability of the designed sequence to accomodate many neutral mutations acknowledged [1] [4, 19 21], the actual quantitative analysis of how many mutations exist that preserve the native state 
was missing.

In what follows we present a quantitative analysis of how many neutral mutations can proteinlike sequences of various degree of gap optimization accomodate. The outcome of this analysis is baffling. In fact, it will be concluded that designed proteins can accomodate billions of multiple mutations without changing their ability to fold on short call into the native conformation.

For the analysis we use lattice model of a protein that has been used earlier by us [18,23, 24, and others [25,26]. The model sequences are composed of aminoacids of 20 types and contain 36 monomers. Two aminoacids are considered interacting if they occupy neighboring positions on the lattice but are not sequence neighbors. The energy of the interaction depends on the identity of the aminoacids involved, so that there is a $20 \times 20$ parameter matrix that describes the energetics in the model. We used the set of parameters suggested by Miyazawa and Jernigan ( table 6 of Ref. 27]). The associated standard deviation of the interaction energies between different aminoacid types is $\sigma=0.3$.

Our approach to protein simulations is based on the idea of designing sequences having a large energy gap in the target conformation chosen to serve as a native state for simulations [4, 28,

A sequence that has sufficiently low energy in a conformation chosen as native is denoted as $\mathrm{S}_{36}$ (cf. caption to Fig. 1). This sequence is the same as was studied in previous publications [18,23,24]. In the units we are considering $\left(R T_{\text {room }}=0.6 \mathrm{kcal} / \mathrm{mol}\right)$, the energy of $\mathrm{S}_{36}$ in its native conformation (cf. Fig. $1(\mathrm{a})$ ) is $E_{\text {nat }}=-16.5$. Starting from a random configuration, the sequence $\mathrm{S}_{36}$ always reaches the native configuration, and it does it in a rather short time, of the order of $10^{6} \mathrm{MC}$ steps. This is a consequence of the fact that the value of the energy gap $\delta(=2.5)$, that is, the energy difference between the native and the lowest dissimilar configuration (configuration with a similarity parameter $q$ [29] much smaller than one) is large, much larger than the variance of the contact energies. The goal of our present analysis is to characterize quantitatively how many mutations can $S_{36}$ tolerate without losing the ability to fold into its native state. In other words, our study aims at 
providing an estimate of the number of sequences, having a certain degree of homology to $S_{36}$, that fold into its native structure.

To characterize quantitatively single or multiple mutations, we ascribe to them a value $\Delta E[18]$, defined as the difference between the energies of the altered sequence $\left(\mathrm{S}_{36}{ }_{36}\right)$ and of the intact chain $\left(\mathrm{S}_{36}\right)$, both calculated in the native configuration (Fig. 1(a)). The quantity $\Delta E$ is a measure of how the energy gap changes upon a mutation provided that the distribution of energies of conformations that are dissimilar to the native state remains unaffected by the mutation [18]. This was shown to be the case when mutations do not change the aminoacid compositions [3, [, 18]. In this study we have analysed both the kinds of mutations which conserve and which do not conserve the composition of the protein. This gives a lower and an upper limit for the number of mutations which the "wild-type" sequence can tollerate.

A complete enumeration of all sequences $\mathrm{S}_{36}^{\prime}$ has been done up to seven mutations keeping fixed the amino acid composition of the chain (swapping), and up to four without this constraint (pointlike). Simulating the dynamics [U] of fifty sequences chosen among the mutated sequences, with the same composition of $\mathrm{S}_{36}$ and with $\Delta E<\delta$, it turned out that in $100 \%$ of the cases, they can reach the native conformation in a time comparable to the folding time of $S_{36}$. Repeating the same analysis on fifty sequences with up to four pointlike mutations, we observed that only in three cases the chain finds conformations dissimilar from the native one, with lower energy, and it is not able to find the native conformation within the simulation time.

Further we studied the impact of pointlike mutations on sequences having different degree of design. To this end we calculated the distributions $n_{2}(\Delta E)$ associated with two pointlike mutations for the case of three sequences designed to fold into the structure shown in Fig.1(a) with different energy gaps. The distributions for all three sequences appear to be very similar to each other (Fig.2). This is also true for composition-conserving mutations and for the different numbers of mutations we have analyzed (data not shown). These results suggest that the distribution $n_{m}(\Delta E)$ associated with $m$ mutations has some degree of universality. 
Given a sequence characterized by an energy gap $\delta$, it is then possible to calculate the number of sequences which fold to the same native structure (i.e. for which there is still some energy gap between the native structure and the bulk of decoys) and which differ from the "wild-type" sequence by $m$ mutations. To do this one has to calculate the quantity

$$
N_{m}(\delta)=\int_{-\infty}^{\delta} d E n_{m}(E) .
$$

As an example we provide the function $N_{m}(\delta)$ for $m=4$ and for the case of pointlike mutations (Fig.3). The corresponding values of $N_{m}$ for the sequence $\mathrm{S}_{36}$ (whose gap is $\delta=2.5)$ for up to 4 pointlike and 7 swap mutations are shown in Table 1 . The calculation of the total number of sequences $N(\delta)=\sum_{m} N_{m}(\delta)$ is beyond our calculational power, and can be established only with approximate methods [30]. The results obtained with the "small" number of mutations shown in Table 1, and which provide a lower limit to the total number of sequences folding to the same native structure is in any case impressive, namely $10^{8}-10^{9}$. The same study has been repeated using other two fully compact target structures (Figs. 1(b) and 1(c) ), generated by the collapse of a 36 monomers homopolymeric chain at low temperature (below the $\theta$-point, see e.g. [31]). The results are virtually identical to the ones shown in Fig. 2.

The present study further suggests that the normalized gap $\xi=\delta / \sigma$ (or the closely related to it z-score [1, 29, 32]) is a major determinant of the ability of sequences to fold. To this end, the "resilience" of sequences against point mutations is directly related to their energetic impact: if the cumulative effect of mutations on the energy of the native state is weak enough so that the energy gap for the native state remains, the mutations are neutral and the mutated sequences will still fold into the native state, albeit at a decreased stability. Therefore, the whole issue of the estimating the number of mutations that are tolerated by a sequence (and hence the number of homologous sequences that fold to the same conformation) is reduced to enumerating mutations that keep the energy gap as defined before.

Another aspect of sequence design known as "designability" was discussed by a number of 
authors [19,33,34. The concept of "designability" focuses on the entropy in sequence space stating that structures that can accomodate more sequences that have them as the nondegenerate ground state are more "designable", and represent the structures of naturally existing proteins. Our study is not entirely unrelated to the issue of designability since it shows that the greater the gap is, the more sequences homologous to the "wild type" sequence exist that target on the "native" conformation in the folding process and, as a consequence, has this conformation as its non-degenerate ground state. However, it addresses in fact a different question, namely: how many sequences homologous to the "wild-type" exist that fold into the same conformation, being this number a lower limit for the degree of designability of a structure. In order to fully address this question within our approach one has to consider two further issues: a) that there exist many non-homologous sequences that can still fold to the same conformation [20,35,36], and b) that the designability principle emphasizes the strong dependence of the number of sequences on the properties of the target structure. In any case, we have provided circumstantial evidence concerning the fact that designability of a given structure may be closely related to the maximal gap with which sequences can be fit into it. (A similar point was also made earlier by several authors $1921,33]$ ). In this case the issue of designability reduces to the question of what structures allow sequences with greater gaps. We are planning to address this issue in the near future.

Summing up, in this paper we provided a quantitative estimate of the number of mutated sequences that are still able to fold to the same conformation and found it to be "astronomically" large. The actual number of these sequences, and thus the designability of the corresponding conformation, is controlled by the dimensionless parameter $\xi=\delta / \sigma$, which in turn also controls the folding ability of the notional protein.

\section{ACKNOWLEDGEMENTS}

This work was partially supported by NIH grant RO1 GM52126 (to ES). Financial support by NATO under grant CRG 940231 is gratefully acknowledged. 


\section{TABLES}

TABLE I. The number of mutated sequences $\mathrm{S}_{36}$ which fold into the native conformation shown in Fig 1(a). In column one the number of mutations $m$ is shown. Columns 2 and 3 are associated with composition conserving results (c.), while columns 3 and 4 correspond to pointlike mutations (n. c.). Columns 2 and 4 display the number of sequences associated with a change in energy $\Delta E$ smaller than the gap $\delta$, while columns 3 and 5 display the total number of sequences associated with the number of mutations $m$.

\begin{tabular}{|l|r|r|r|r|}
\hline \hline $\mathrm{m}$ & $\Delta E<\delta(\mathrm{c})$. & Tot $(\mathrm{c})$. & $\Delta E<\delta($ n.c. $)$ & Tot (n.c.) \\
\hline 1 & & & 613 & 684 \\
\hline 2 & 447 & 630 & $1.59 \cdot 10^{5}$ & $2.27 \cdot 10^{5}$ \\
\hline 3 & 3339 & 14280 & $2.30 \cdot 10^{7}$ & $4.89 \cdot 10^{7}$ \\
\hline 4 & $1.37 \cdot 10^{5}$ & $5.30 \cdot 10^{5}$ & $1.99 \cdot 10^{9}$ & $7.68 \cdot 10^{9}$ \\
\hline 5 & $4.29 \cdot 10^{5}$ & $3.39 \cdot 10^{6}$ & & \\
\hline 6 & $2.53 \cdot 10^{7}$ & $5.14 \cdot 10^{8}$ & & \\
\hline 7 & $2.78 \cdot 10^{8}$ & $1.55 \cdot 10^{10}$ & & \\
\hline \hline
\end{tabular}




\section{FIGURES}

FIG. 1. (a) Conformation onto which the sequence $S_{36} \equiv$ SQKWLERGATRIADGDLPVNGTYFSCKIMENVHPLA has been designed. In (b) and (c) we display two other fully compact target structures, used also as natives (for another sequences). These conformations were generated by collapsing a homopolymeric chain at low temperature.

FIG. 2. Distribution $n_{2}(\Delta E)$ associated with two pointlike mutations, carried out in three different sequences, with gaps $\delta=1.3$ (dashed curve), $\delta=1.6$ (continuous curve) and $\delta=2.5$ (dotted curve) respectively. The three "root" sequences display no appreciable similarity.

FIG. 3. Number of sequences which fold into the conformation shown in Fig.1(a) and obtained from all possible four-aminoacid pointlike mutations of $\mathrm{S}_{36}$ that still preserve the gap. 


\section{REFERENCES}

[1] R.Goldstein, Z.A. Luthey-Schulten, and P.Wolynes. (1992) Proc. Natl. Acad. Sci. USA 89, 4918-4922.

[2] A.Sali, E.I.Shakhnovich, and M.Karplus. (1994) Journ. Mol. Biol. 235, 1614-1636.

[3] E.Shakhnovich and A.Gutin. (1993) Proc.Natl. Acad. Sci. USA 90, 7195-7199.

[4] E.I.Shakhnovich. (1994) Phys.Rev.Lett. 72, 3907-3910.

[5] S. Govindarajan and R.Goldstein. (1995) Biopolymers 36, 43-51.

[6] A.Gutin, V.Abkevich, and E.Shakhnovich. (1996) Phys Rev Lett 77, 5433.

[7] J.Bryngelson, J.N.Onuchic, N.D.Socci, and P.Wolynes. (1995) Proteins: Struct. Funct. and Genetics 21, 167-195.

[8] E.I.Shakhnovich. (1997) Curr. Opin. Struct. Biol. 7, 29-40.

[9] K. Gast, A.F. Chafotte, D.Zirwer, Y.Guillou, M.Mueller-fromme, C.Cadieux, M.Hodges, G.Damaschun, and M.Goldberg. (1997) Protein Science 6, 2578-2588.

[10] A.Davidson and R.Sauer. (1994) Proc. Natl. Acad. Sci. USA 91, 2146-2150.

[11] G. Makhatadze and P.L.Privalov. (1995) Adv.Prot.Chem. 47, 307-425.

[12] S.E.Jackson and A.R.Fersht. (1991) Biochemistry 30, 10428-10435.

[13] J.D.Bryngelson. (1994) J.Chem.Phys. 103, 6038-6045.

[14] V.Pande, A.Yu. Grosberg, and T.Tanaka. (1995) J.Chem.Phys. 103, 1-10.

[15] A.P. de Araujo and T.Pochapsky. (1996) Folding $\&$ Design 1, 299-314.

[16] E.I.Shakhnovich and A.M.Gutin. (1991) J.Theor.Biol. 149, 537-546.

[17] T.Creighton. Proteins. Structure and Molecular Properties. W.H.Freeman \& Co, N.Y., (1992). 
[18] G.Tiana, R.Broglia H.E.Roman, E.Vigezzi, and E.I.Shakhnovich. (1998) J.Chem.Phys. 108, $757-761$.

[19] S. Govindarajan and R.Goldstein. (1995) Proc. Natl. Acad. Sci. USA 93, 3341-3345.

[20] E.I.Shakhnovich. (1998) FoldingÉSDesign 3, R45-R58.

[21] M.Vendruscolo. (1998) Physica A 249, 576-580.

[22] HJ Bussemaker, D.Thirumalai, JK Bhattacharjee (1997) Phys.Rev.Lett. 79, 3530-3533.

[23] V.Abkevich, A. Gutin, and E.Shakhnovich. (1994) Biochemistry 33, 10026-10036.

[24] V.Abkevich, A. Gutin, and E.Shakhnovich. (1994) J.Chem.Phys 101, 6052-6062.

[25] N.Socci, W.Bialek, and J.Onuchic. (1994) Phys.Rev.E 49, 3440-3443.

[26] D.Klimov and D.Thirumalai. (1996) Phys.Rev.Lett 76, 4070-4073.

[27] S.Miyazawa and R.Jernigan. (1985) Macromolecules 18, 534-552.

[28] E.Shakhnovich, V. Abkevich, and O.Ptitsyn. (1996) Nature 379, 96-98.

[29] A. Gutin, V.Abkevich, and E.Shakhnovich. (1995) Proc Natl. Acad. Sci. USA 92, 12821286.

[30] R. A. Broglia, G. Tiana and H. E. Roman, (to be published)

[31] I. Lifshitz, A. Grosberg, A. Khokhlov, Rev. Mod. Phys. 50, 683 (1978)

[32] J.U.Bowie, R.Luthy, and D.Eisenberg. (1991) Science 253, 164-169.

[33] A.V.Finkelstein, A.Gutin, and A.Badretdinov. (1995) Proteins: Struct. Function genetics 23, 142-149.

[34] H.Li, N.Wingreen, and C.Tang. (1996) Science 273, 666-669.

[35] L.Holm and C.Sander. (1993) J.Mol.Biol 233, 123-138. 
[36] E.Shakhnovich and A.Gutin. (1993) Protein Engineering 6, 793-800. 

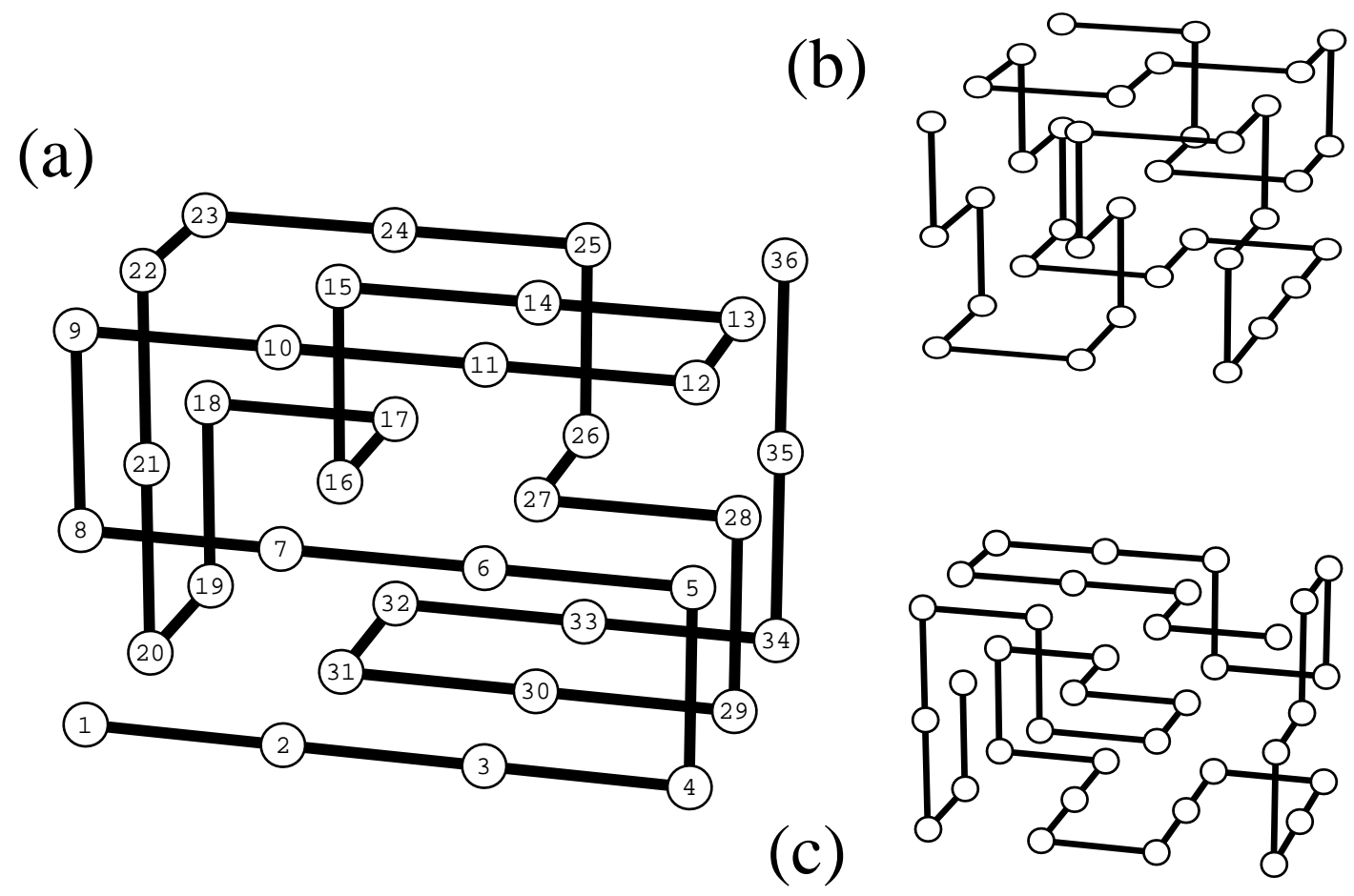


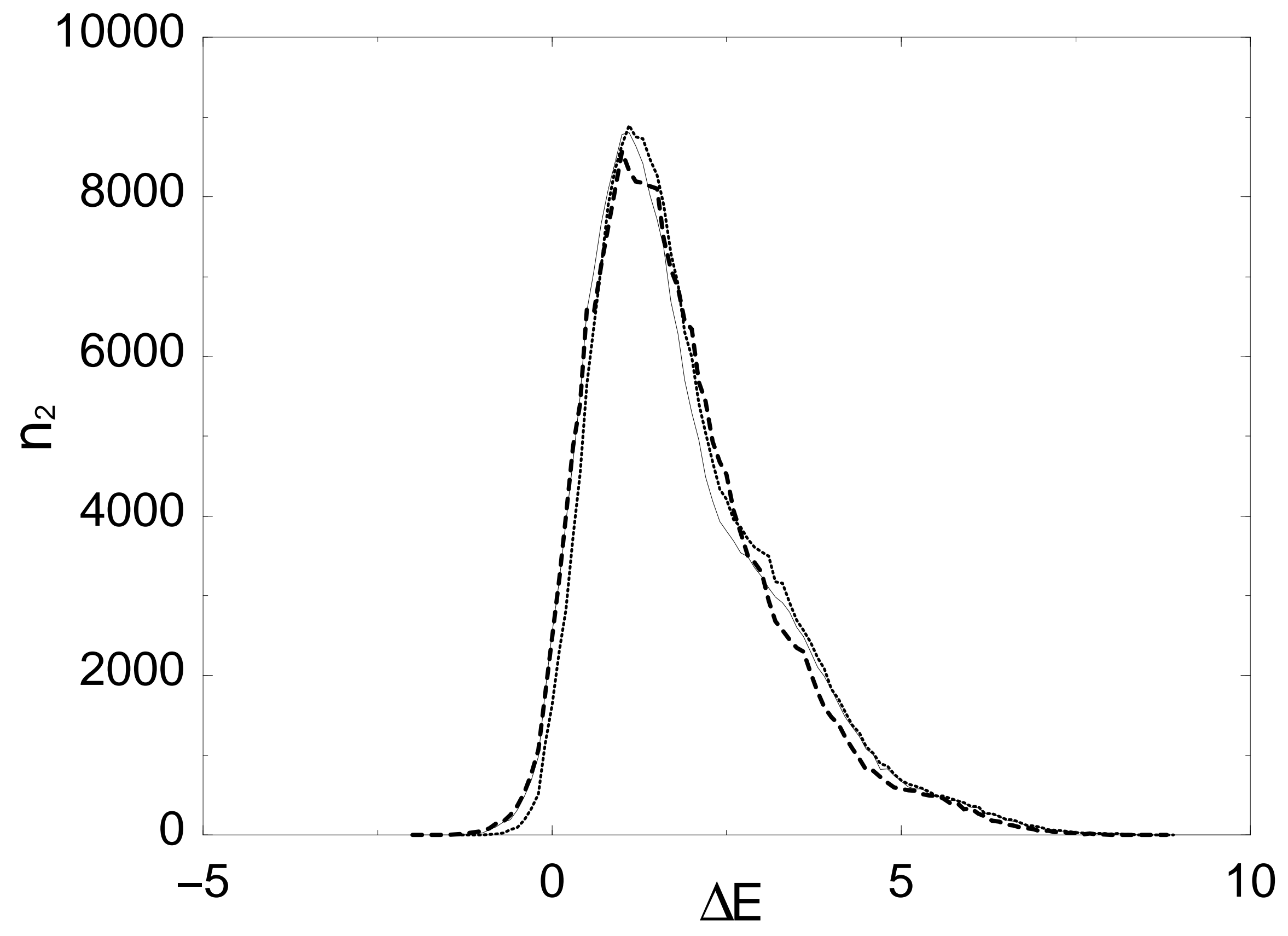




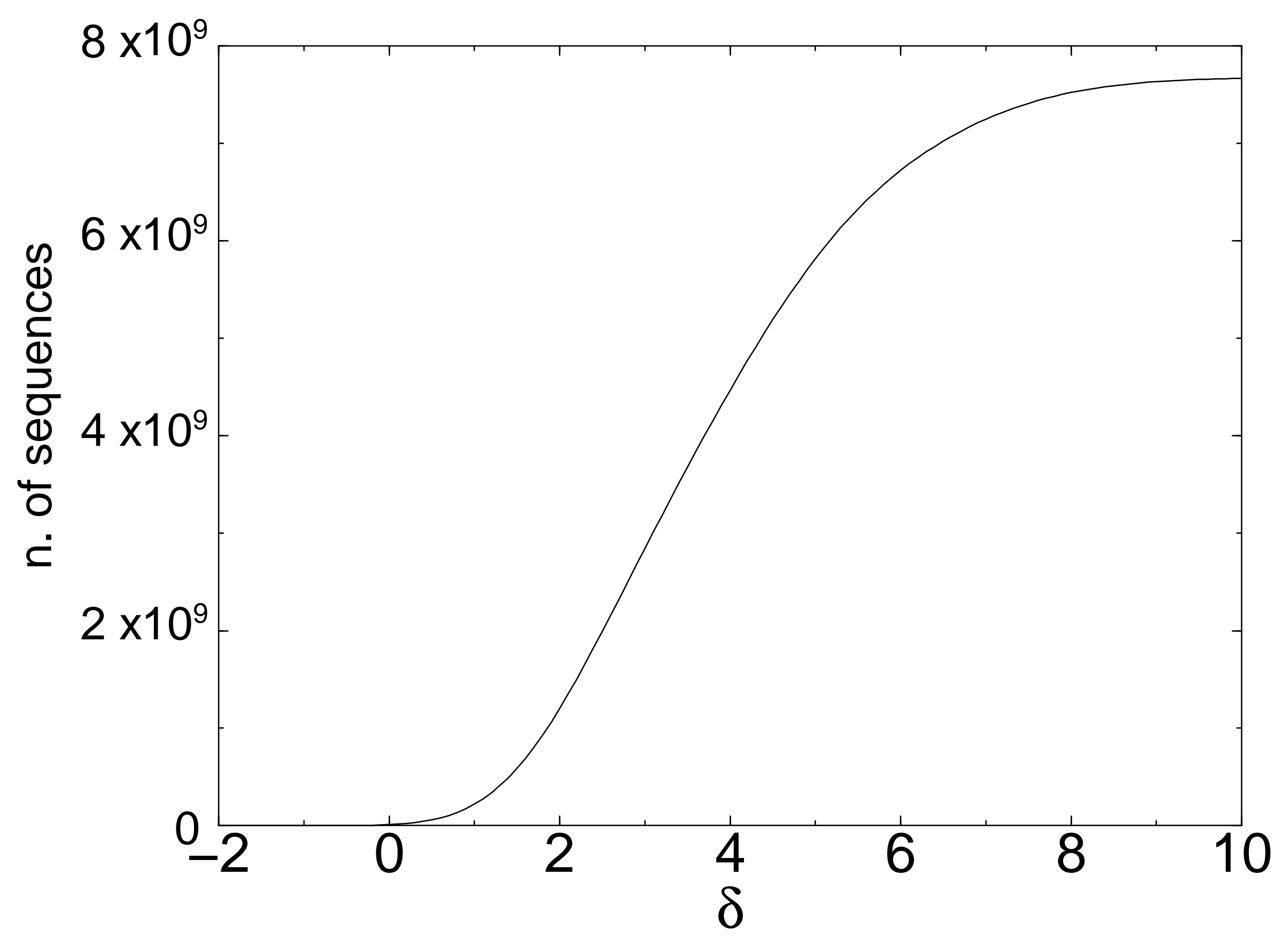

